\title{
Cambio constitucional y capacidades institucionales: un análisis de la protesta social en Colombia
}

\author{
Marcela Velasco Jaramillo \\ Universidad de los Andes, Colombia
}

Resumén: El trabalho examina por que la Reforma Constitucional de 1997 en Colombia, disenada para reducir los efectos negativos del centralismo político no sossegó la comtestacion social. El trabajo analisa bases de datos para una serie de tiempo y estadísticas variadas

Palabras-clave: contestación social, Colombia, ciudadania, Estado

Abstract: The article examines why the Consitutional Reform of 1991 in Colombia, which was elaborated to reduce the negative impact of the political centralism, did not succed to calm social protests. Time series data and various statistics are used.

Key words: social protest, Colombia, citizenship, State 
JARAMILLO, M.V. Cambio institucional e incapacidades institucionales: un análisis...

La política contestataria se enfoca en el estudio de la política transgresiva, o aquellas acciones colectivas y públicas que en general desafían el orden político establecido para exigir el reconocimiento de actores excluidos, la ampliación de derechos o la redefinición de categorías políticas (MCADAM et al, 2001) ${ }^{1}$. El énfasis en la política contestataria permite una compresión más compleja de la relación entre Estado y sociedad, ya que dicha relación va más allá de las normas formales de hacer política como son las elecciones, el cabildeo o los procesos parlamentarios. Los procesos de contestación además ponen en juego al poder, el mérito y los recursos, los cuales están distribuidos de manera no proporcional en la sociedad (RUESCHEMEYER et al, 1992; ECKSTEIN, 1989), y cuyo acceso asegura la auto determinación, y una buena vida libre de necesidades. La falta de poder, la incapacidad, las desventajas y la pobreza se correlacionan con la dependencia, la miseria, la necesidad y el abuso. Por lo tanto, la gente hace reclamos para aumentar su poder, mérito, capacidades y recursos.

El presente trabajo se enmarca en la literatura de la política contestataria y examina por qué la reforma constitucional de 1991, ostensiblemente diseñada para reducir los efectos negativos del centralismo político en la democracia, no sosegó la contestación social en Colombia (o las huelgas, protestas y tomas Ilevadas a cabo por trabajadores, residentes urbanos, campesinos y estudiantes). Esta Constitución trató de darle una respuesta institucional a la oleada de descontento sociopolítico de los años ochenta, e incrementó el número de mecanismos formales de representación y participación política como el plebiscito, el referendo, la tutela, las acciones populares, entre otros. Teóricamente, estos mecanismos no sólo servirían para mejorar la relación entre Estado y ciudadanos, sino también para aminorar el uso de la protesta, e inclusive, de la violencia para presionar al Estado. Sin embargo, las protestas continuaron, e inclusive, se incrementaron hacia finales de la década, lo que es particularmente preocupante ya que protestar en Colombia conlleva altos costos para la vida, propiedad y libertad de los manifestantes. Todo esto sugiere que para acoger las demandas de mayor participación y disuadir la política contestataria no basta con democratizar las instituciones políticas. Por lo tanto, necesitamos evaluar factores adicionales que puedan explicar la contestación en Colombia.

La literatura especializada sobre los movimientos sociales en Colombia afirma que la protesta es el producto de los efectos negativos de la centralización política (SANTANA, 1983; LEAL 1991), de la debilidad de la sociedad civil (ARCHILA, 2003c) de un reto a las categorías políticas que justifican la exclusión de grupos enteros

\footnotetext{
${ }^{1}$ Según McAdam, Tarrow y Tilly (2001) la política contestataria es una “interacción episódica, pública y colectiva entre reclamantes y los objetos de su reclamo cuando (a) por lo menos un gobierno es reclamante u objeto de reclamo y (b) si se lograra el reclamo, se verían afectados los intereses de por lo menos una de las partes".
} 
como portadores de derechos (RAMÍREZ, 2002), y de la ineficiencia de los mecanismos de representación, que deja a los ciudadanos con la sola opción de utilizar la protesta para influenciar al sistema político. Otros estudios plantean que las protestas responden a necesidades materiales, como la pobreza, la falta de tierra, y los bajos ingresos, todo esto empeorado por las contradicciones de clase y la concentración de la riqueza y del poder. Así, los actores contestatarios impugnan las condiciones de dependencia, de pobreza y de subdesarrollo que deterioran su bienestar socioeconómico (GILHODES, 1970; PÉCAUT, 1982; ZAMOSC, 1989).

No obstante la variedad de enfoques arriba citados, esta literatura no le ha prestado suficiente atención a la relación entre la protesta y el desarrollo de instituciones como la ciudadanía y el Estado, y tampoco ha hecho buen uso de los métodos estadísticos para establecer relaciones de causalidad entre la protesta y los factores que la explican. Por lo tanto, el trabajo evalúa la relación entre la protesta y el desarrollo de estas instituciones mediante el análisis estadístico y propone que la protesta social resulta de la pérdida de capacidades del Estado para contener la protesta y/o proteger el bienestar y los derechos de las personas, y del deterioro de las capacidades ciudadanas para acceder a recursos políticos y económicos que les permita influenciar las políticas públicas. De forma más precisa, este trabajo evalúa tres hipótesis en el periodo de tiempo entre 1964 y 2000:

(1) La contestación social aumenta en la medida en que se deterioran las capacidades ciudadanas y las del Estado. La pérdida de capacidades les genera inseguridad socioeconómica y política a los miembros del sistema político. Además, si los mecanismos y recursos legales para hacer demandas son inefectivos, los ciudadanos utilizarán mecanismos contestatarios para presionar al Estado.

(2) En un contexto de deterioro de las capacidades ciudadanas y del Estado, el paso del tiempo aumenta la contestación social. En la medida en que pasa el tiempo, el uso de los mecanismos contestatarios se institucionaliza o se vuelve rutinario si el estado sigue siendo débil y las capacidades ciudadanas son inoperantes.

(3) La Constitución de 1991 no afectó el comportamiento de la política contestataria. Los cambios constitucionales toman mucho tiempo en alterar comportamientos políticos que responden a procesos de mayor escala, como son la construcción del Estado o la democratización. Estos procesos macro-sociales están inmersos en dinámicas nacionales e internacionales que pueden anular los efectos positivos de un cambio institucional o distorsionar las funciones institucionales. 
JARAMILLO, M.V. Cambio institucional e incapacidades institucionales: un análisis...

Estas hipótesis se examinarán estadísticamente utilizando el método de regresión de series de tiempo. Las series de tiempo de contestación se toman de las bases de datos de protestas del Centro de Investigación para la Educación Popular (CINEP) y las de ciudadanía y Estado de las bases de datos del Departamento de Planeación Nacional, el Departamento Administrativo Nacional de Estadística, el Banco de la República y el Banco Mundial.

La capacidad del Estado supone procesos de monopolización de la fuerza, y de control de recursos, actividades y personas (MCADAM et al, 2002; HUBER, 1995, SKOCPOL, 1985). Dicha consolidación se ve afectada por los intereses de las elites políticas, y está condicionada por procesos locales, nacionales e internacionales (RUESCHMEYER et al, 1991). Además, las capacidades de los Estados con altos niveles de deuda externa, déficit fiscal, economías débiles y que además están reestructurando sus instituciones son fuertemente condicionadas por actores internacionales (WEISS, 2003), ya que las elites estatales les ofrecen más privilegios a los inversores internacionales, y siguen directrices políticas de países extranjeros u organizaciones financieras multinacionales (VILAS, 1995). El proceso de consolidación del Estado está limitado por la contestación. Sobre este tema los institucionalistas históricos argumentan que si bien las instituciones políticas ${ }^{2}$ condicionan y proveen orden al comportamiento político, éstas cambian como resultado del conflicto (STEINMO and THELEN, 1992). Las instituciones distribuyen poder y "emergen no de una preocupación compartida por lograr ganancias mutuas por medio de la cooperación, sino que surgen del conflicto político y la negociación estratégica entre actores sociales" (THELEN, 2003, p. 215). Como el resultado del conflicto produce balances institucionales favorables para los actores más poderosos (THELEN, 2003, p. 216), los actores contestatarios optan por ignorar las instituciones prevalecientes para así tener un impacto sobre la política (PIVEN and CLOWARD, 1977).

Por otro lado, la capacidad ciudadana contempla las habilidades de la población para ejercer derechos y tener bienestar. En una crítica al argumento de T.H. Marshall sobre los derechos ciudadanos, Bottomore (1998) contradice la descripción que presenta Marshall (1950) del desarrollo de los derechos civiles, políticos y sociales como producto de la evolución política, y plantea que el compromiso público (del Estado) con la ciudadanía se logró por medio de acciones contestatarias y sobretodo después de que una clase social lograra transformar las instituciones a su favor (BOTTOMORE, 1992) ${ }^{3}$. Por lo tanto, la ciudadanía es una

\footnotetext{
2 Las instituciones son el conjunto de reglas, normas y tradiciones formales e informales y las organizaciones y regulaciones sociopolíticas relativamente establecidas que condicionan las interacciones políticas. Estas reglas y organizaciones son afectadas por factores socioeconómicos y por la cultura política, y también por acontecimientos históricos significativos.

${ }^{3}$ Así, la burguesía obtuvo derechos civiles contestando el poder de la monarquía y lograron los derechos a la propiedad, la vida y la libertad. Los trabajadores y otros grupos subalternos protestaron para ganar
} 
institución que se moldea a través de la contestación, y los derechos de los ciudadanos sólo son significativos si un Estado con capacidades los confirma.

Por otro lado, el tener derechos aumenta las capacidades de lograr las cosas que la gente valora, como tener educación o participar en la toma de decisiones (SEN, 1999). Pero, para lograr esas cosas los individuos necesitan recursos pues los derechos restringidos por la pobreza no son significativos. Según Sen (1999) cinco mecanismos privados y públicos se mezclan para promover las libertades y capacidades (capabilities) de las personas: (1) las libertades políticas proporcionan los derechos esenciales de las instituciones poliárquicas, (2) los servicios económicos le ofrecen a la gente las oportunidades de usar sus recursos para producir, consumir e intercambiar, (3) las oportunidades sociales como el acceso a la educación y a los servicios de salud mejoran la participación en los asuntos públicos, (4) las garantías de transparencia ofrecen acceso claro y veraz a la información y previenen la corrupción, y (5) la seguridad protectiva provee beneficios de desempleo y otros subsidios para aliviar las necesidades de aquellos afectados por las crisis económicas profundas.

En Colombia, seis procesos más o menos interrelacionados desalientan la consolidación de los mecanismos que lista Sen y debilitan las capacidades ciudadanas: (1) las presiones internacionales para implementar medidas neoliberales recesivas, (2) la violencia centrífuga, (3) la influencia de intereses particulares y sobretodo empresariales (nacionales e internacionales) sobre las instituciones democráticas, (4) la democracia delegativa, (5) el mal desempeño económico, y (6) la debilidad de la sociedad civil. En primer lugar, las reformas del Estado estuvieron dirigidas a reducir la intervención del Estado en la prestación de servicios sociales. Estas políticas han tenido efectos negativos sobre el alivio de la pobreza y la distribución más igualitaria de la riqueza. Segundo, el aumento de la violencia en todos los niveles sociales y políticos ha polarizado la sociedad y ha fracturado las organizaciones ciudadanas. Tercero, una versión criolla de la "ley de oro" (the golden rule) de Ferguson (1995) ${ }^{4}$ se puede observar. Aquí, las organizaciones que representan intereses privados, de sectores financieros y de importadores ejercen poder directo sobre el proceso político no sólo por medio de las elecciones, sino también por medio del uso privilegiado del lobby, las campañas en los medios de comunicación, el clientelismo y la corrupción.

En cuarto lugar, la concentración de la toma de decisiones en la rama ejecutiva menoscaba las instituciones representativas y caracteriza la democracia

derechos políticos y sociales como el sufragio, la libertad de asociación y los seguros sociales. Bottomore argumenta entonces que las coaliciones gobernantes que representan intereses empresariales restringen los derechos ciudadanos a los civiles y políticos; y las coaliciones laboristas apoyan los derechos sociales y el aumento del gasto público para aumentar beneficios sociales (Bottomore 1992: 121).

4 Donde los grupos más prósperos del sistema político americano aumentan su influencia sobre la política pública inyectándole capital a las campañas electorales. 
JARAMILLO, M.V. Cambio institucional e incapacidades institucionales: un análisis...

colombiana como un sistema delegativo ${ }^{5}$ menos liberal, donde las políticas gubernamentales no se acercan a las promesas electorales, y los líderes esperan que los votantes sean pasivos. O'Donnell (1994) argumenta que en las democracias delegativas la política pública sigue un patrón errático y la sociedad protesta las políticas anti-populares. Si es necesario, las protestas populares son reprimidas violentamente. En quinto lugar, el desempleo y el deterioro de los salarios reducen los recursos de movilización y organización de los grupos marginados de la política.

La contestación social, la capacidad del Estado y las capacidades ciudadanas

La contestación de los trabajadores, los pobres urbanos, los campesinos y los estudiantes expande el alcance y el significado de la ciudadanía. Estos actores defienden derechos sociales y políticos previamente adquiridos, y buscan reformar y democratizar el Estado. Aunque muchos actores contestatarios promulguen discursos en contra del gobierno y se conviertan en víctimas de la represión del Estado, estos reconocen al Estado como una institución estratégica para formalizar derechos y reclamos. Por su lado, las elites estatales responden a los episodios de protesta democratizando las instituciones, ignorando las demandas, o reprimiendo a los actores sociales. La negociación lleva a la democratización y la expansión de la ciudadanía, mientras que la represión lleva al autoritarismo. Este trabajo denomina "protesta social" las acciones colectivas contestatarias de trabajadores, campesinos, estudiantes y pobres urbanos, entre otros, para distinguirla de la contestación guerrillera o paramilitar ${ }^{6}$ prevaleciente en Colombia.

La serie de tiempo de contestación social fue tomada de las bases de datos del Centro de Investigación para la Educación Popular (CINEP) que recoge información anual sobre protestas sociales. Esta información ha sido publicada por Archila (2003c) quien presentó estadísticas de protestas de trabajadores, pobres urbanos, estudiantes, campesinos y otros actores entre 1958 y 1974. Los datos de los años 1975-2000 fueron publicados por Delgado (2003) quien muestra información sobre huelgas laborales, Prada (2003) sobre campesinos, y García (2003a, 2003b, 2003c) sobre pobres urbanos, estudiantes y otros actores.

Ya que los conceptos de capacidad del Estado y de la ciudadanía pueden ser evaluados con una amplia gama de indicadores empíricos - como se demostrará

\footnotetext{
5 Sistemas de gobiernos mayoritarios e individualistas que concentran el poder en la oficina del presidente.

6 Otros tipos de política contestataria en Colombia incluyen el conflicto intra-gubernamental donde funcionarios del estado usan mecanismos de protesta, desobediencia o resignación a sus cargos con el fin de presionar otra rama del gobierno. Los funcionarios públicos (especialmente de la rama ejecutiva) han usado estrategias extra-constitucionales (Ej. el terror o las acciones paramilitares) para derrotar una clase social o un sector de la economía, prevenir el cambio social, o reformar el estado.
} 
abajo - he decidido agrupar los indicadores que sirven para medir cada concepto en un número más pequeño de factores. El análisis factorial es un procedimiento estadístico que identifica la correlación entre un gran número de indicadores relacionados entre sí y los agrupa en un conjunto más pequeño de índices. Así, con la ayuda de la función de SPSS ${ }^{7}$ para hacer análisis factorial, se crearon índices que permiten medir los conceptos de capacidades ciudadanas y del Estado.

Los siguientes criterios guiaron la selección de las variables para medir cada concepto: las estadísticas que valoran las libertades políticas y el bienestar socioeconómico de las personas (i.e. esperanza de vida, educación, salarios) fueron utilizadas para medir la capacidad ciudadana. La capacidad del Estado se mide con estadísticas macroeconómicas internas y externas, y con indicadores que miden el control del Estado sobre personas y recursos en su territorio.

Las capacidades del Estado ${ }^{8}$

Las estadísticas utilizadas para medir las capacidades financieras y de control de actividades del Estado incluyen el porcentaje del presupuesto nacional que viene del cobro de impuestos entre 1958 y 2000 (Fuente: Departamento Nacional de Planeación (DNP) 1998, 2002), el déficit fiscal como porcentaje del PIB entre 1958-2000 (Fuente: DNP 1998, 2002), y el índice de inflación entre 1958 hasta 2000 (Fuente: DNP 1998, 2002). El déficit fiscal constituye un problema para la capacidad del Estado ya que se financia con deuda, y el Estado se ve cada vez más obligado a seguir recetas del Fondo Monetario Internacional para reducirlo. Esto limita la autonomía política del gobierno. La tasa de inflación establece si el gobierno está aplicando políticas monetarias expansionistas o restrictivas. Las políticas monetarias expansivas son positivas si redistribuyen el ingreso para generar empleo e incrementar los ingresos de los pobres. Pero si se salen de control pueden tener efectos negativos sobre el crecimiento económico cuando se pierde la confianza en el mercado financiero y se reduce la inversión. En este trabajo, un índice bajo de impuestos como porcentaje del presupuesto, un déficit fiscal alto y un índice alto de inflación se relacionan con la reducción de las capacidades del Estado.

Las estadísticas utilizadas para medir el control de actividades evalúan la habilidad del Estado para proteger las propiedades y las libertades de las personas que viven en su jurisdicción. Esto se puede observar con la serie de secuestros entre 1964 y el 2000 (Fuente: DNP 1998, 2002). El secuestro es un fenómeno que afecta sobretodo a los colombianos más ricos o a los miembros de las fuerzas armadas

\footnotetext{
7 Programa de análisis estadístico para ciencia social.

8 Uma discusion más amplia de estos factores se presenta em la tesis de doctorado que lê estoy presentando al departamento de ciência política de la Universidad de Boston.
} 
JARAMILLO, M.V. Cambio institucional e incapacidades institucionales: un análisis...

del Estado, y su incremento genera un ambiente de inseguridad que afecta la economía y que puede aumentar la fuga de capitales. Si una de las principales funciones del Estado es (como mínimo) proveer protección de las vidas y propiedades de los más ricos ${ }^{9}$, entonces el incremento de los secuestros es una medida próxima de incapacidad del Estado.

En teoría un Estado con capacidades le debe dar respuesta a las demandas de política pública de su población. Las demandas más importantes del pueblo colombiano son el desarrollo económico (i.e. crecimiento económico, servicios públicos y sociales, educación, salud, infraestructura, vivienda) y el fin del conflicto armado (i.e. seguridad y justicia). Estas capacidades se miden con el crecimiento anual de Producto Interno Bruto entre 1958-2000 (WORLD BANK, 2002), y con el gasto social, el gasto en justicia y seguridad, y el gasto en infraestructura como porcentajes del total del gasto público entre 1958-2000 (DNP, 1998; 2002). Índices altos de crecimiento, y porcentajes altos de gasto público son señales de mayor capacidad del Estado.

Finalmente, el desarrollo de Colombia y de su Estado está restringido por el acceso a recursos y mercados internacionales que no siempre se presentan en buenas condiciones para el país. Los constreñimientos internacionales fueron medidos con la deuda externa entre 1958 y 2000 (DNP, 1998; 2002), la ayuda internacional como porcentaje del PIB entre 1960 y 2000, y la balanza externa como porcentaje del PIB entre 1958 y el 2000 (WORLD BANK, 2002). Una deuda externa alta y un porcentaje alto de ayuda extranjera se relacionan con una reducción de capacidades, mientras que un porcentaje alto en la balanza externa se relaciona con un aumento de capacidades.

Las capacidades ciudadanas

Las estadísticas utilizadas para medir el bienestar socioeconómico de los ciudadanos incluyen la tasa de desempleo y los salarios. Estas son estadísticas importantes, pues la población desempleada tiene mayores posibilidades de caer en la pobreza y perder seguridad económica. Los indicadores utilizados incluyen la taza de desempleo ${ }^{10}$ entre 1963 y 2000 (DNP, 1998; 2002); el salario mínimo urbano $^{11}$ entre 1958 y 2000 (DNP, 1998; 2002); y la esperanza de vida al nacer entre 1958-2000 (WORLD BANK, 2002). Un nivel alto de desempleo reduce las

\footnotetext{
9 Tilly (1985) argumenta que los estados se consolidan cuando le dan protección a sus "clientes" internos, o aquellos que pagan impuestos y motivan el crecimiento económico.

10 La serie usa la tasa de desempleo de las siete principales ciudades de Colombia. Como el desempleo se mide mensualmente, utilicé el indicador de septiembre ya que es el mes que citan la Organización Internacional del Trabajo y la Cepal para observar el desempleo en Colombia.

11 Se dividió el salario nominal de cada año (que no corrige la inflación) por el índice de precios al consumir de cada año, y luego se multiplicó la cifra por 100.
} 
capacidades, mientras que el incremento de los salarios y una mejor esperanza de vida al nacer se relacionan con un incremento de las capacidades de los ciudadanos.

Las estadísticas que miden las condiciones que afectan las posibilidades que tienen los ciudadanos para asociarse y presionar la política pública incluyen la escolaridad y la violencia. El número promedio de años de escolaridad entre 1958 . 2000 nos da una idea general del tiempo de participación de los colombianos en el sistema educativo, donde reciben información general sobre instituciones políticas como el voto y los derechos ciudadanos, y aprenden a leer y a escribir (DNP 1998, 2000). La tasa de homicidios incluye asesinatos que resultan del crimen común, el tráfico de drogas y la violencia sociopolítica y se usa como un indicador amplio que mide las manifestaciones económicas, políticas y sociales de la violencia (tasa de homicidios por cada 100,000 desde 1964-2000, DNP 1998, 2002).

Como indicadores de derechos políticos utilizaré dos variables dicotómicas: (1) la implementación de las políticas de elección popular de alcaldes en 1986 y (2) la aprobación del mecanismo de la tutela en 1994 que amplió los recursos legales para reclamar derechos constitucionales $(0=a$ los años cuando la reforma no se había implementado y 1 =a los años después de la implementación de la reforma). Un puntaje igual a 1 en las variables de elección local de alcaldes y de tutela se relaciona con mayores capacidades ciudadanas, una estadística alta en la taza de homicidios apunta a una reducción de capacidades, y un promedio alto de años de escolaridad se relaciona con un incremento de las capacidades ciudadanas.

Los factores

Se hizo un análisis de componentes principales usando análisis factorial con solución de iteraciones ${ }^{12}$ con 11 variables de capacidad del Estado (ver la Tabla 1) y con 7 variables de capacidad ciudadana (Tabla 2).

Una rotación varimax generó la máxima distinción entre los factores al aislar las dimensiones independientes entre las variables. Este ejercicio produjo 2 factores para la capacidad del Estado. Seis indicadores se agruparon en el componente 1 (sus cargas están agrupadas en el cuadro superior izquierdo), y los otros cinco indicadores se cargaron en el componente 2 (cuadro derecho inferior). El componente 1 fue denominado el "índice de capacidades fiscales y jurisdiccionales" (o índice fiscal) ya que las variables que se agruparon en este componente tienen en común que en su mayoría miden las restricciones de presupuesto, económicas y de control de actividades del Estado. El componente 2 fue denominado "índice de

\footnotetext{
12 Principal factoring with iterations solution.
} 
capacidad monetaria y de política pública" (o índice de política pública) porque se agruparon estadísticas de gasto público con el índice de inflación ${ }^{13}$.

\section{Tabla 1. Cargas de las variables de capacidad del Estado en dos factores (Colombia 1964-2000) Matriz de rotación de componentes*}

\begin{tabular}{|c|c|c|}
\hline Variables & $\begin{array}{c}\text { Componente } 1 \\
\text { Capacidades } \\
\text { fiscales y } \\
\text { jurisdiccionales } \\
\text { (índice fiscal) }\end{array}$ & $\begin{array}{c}\text { Componente } 2 \\
\text { molítica pública dica de política } \\
\text { pública) }\end{array}$ \\
\hline
\end{tabular}

Medida reversada de la deuda externa

Medida reversada del número de secuestros

Déficit del gobierno central

Crecimiento del PIB

Medida reversada de ayuda extranjera como \% del PIB

$\%$ del presupuesto del gobierno que viene de cobrar impuestos

Medida reversada de la tasa de inflación

$\%$ gasto del gobierno en infraestructura como total del gasto

$\%$ gasto del gobierno en justicia y seguridad

\begin{tabular}{|l|l|}
\hline 0.91 & \\
0.91 & \\
0.82 & -0.26 \\
0.75 & \\
-0.63 & -0.58 \\
0.52 & -0.36 \\
& 0.90 \\
& 0.81 \\
-0.41 & 0.79 \\
& -0.76 \\
& -0.59 \\
\hline
\end{tabular}

$\%$ gasto social

Balanza externa como \% del PIB

32.40

Total de la variación que explica cada componente

34.50

Método de extracción: Análisis de componentes principales. Método de rotación: Varimax con normalización Kaiser. ${ }^{*}$ La rotación convergió e 3 iteraciones.

Con respecto a las capacidades ciudadanas, el análisis factorial de 7 variables produjo 2 componentes. Sin embargo, el segundo componente será excluido del análisis pues sólo explica $14.8 \%$ de la variación entre las variables.

\footnotetext{
13 Para simplificar el análisis, un número de variables fueron re-codificadas para cambiar su dirección, así un número alto siempre se relaciona con un aumento de capacidades ciudadanas o del estado. Las variables re-codificadas han sido denominadas "medidas reversadas" del indicador original. Para cambiar su dirección se hizo una sustracción de todos los casos anuales del indicador más alto de la serie. Por ejemplo, en la serie de secuestros el año 1999 registra 3706 secuestros. Como supongo que un alto número de secuestros significa un deterioro de capacidades estatales, la dirección de este indicador debía ser cambiada. Para producir el "indicador reversado" se hizo una sustracción de todos los casos de la serie del indicador más alto (3706 - la serie de secuestros = medida reversada). Esto convirtió 3706 en 0 , el punto más bajo para las capacidades del estado en la serie de "secuestros reversada". Al mismo tiempo, el indicador más bajo de secuestros en la serie original (44 secuestros en 1979) se convirtió en 3662, o el punto más alto para las capacidades del estado en la serie reversada.
} 
Tabla 2. Cargas de las variables de capacidad ciudadana en dos factores (Colombia 1964-2000) Matriz de rotación de componentes*

\begin{tabular}{|c|c|c|}
\hline Variables & $\begin{array}{c}\text { Componente } 1 \\
\text { Capacidad } \\
\text { ciudadana }\end{array}$ & Componente 2 \\
\hline Medida reversada de la tasa de homicidios & -0.96 & \\
\hline Elección local de alcaldes & 0.93 & \\
\hline Escolaridad (promedio nacional) & 0.92 & \\
\hline Expectativa de vida al nacer & 0.91 & \\
\hline Implementación de la tutela & 0.81 & -0.24 \\
\hline Salario mínimo urbano & .0 .77 & \\
\hline Medida reversada de la tasa de desempleo & & 0.99 \\
\hline Total de la variación que explica cada componente & 67.79 & 14.79 \\
\hline
\end{tabular}

Método de extracción: Análisis de componentes principales. Método de rotación: Varimax con normalización Kaiser. *La rotación convergió e 3 iteraciones.

\section{El paso del tiempo y la Constitución de 1991}

La variable del paso del tiempo se mide de la siguiente forma, 1964 es el primer año y obtiene un 1, 1965 obtiene un 2, y así sucesivamente hasta el 2000, el último año de la serie que obtiene un 37. La Constitución de 1991 se mide como una variable dicotómica es decir, obtiene un 1 entre 1991 y 2000 cuando la reforma se empezó a implementar, y un 0 entre los años 1964 y 1990 cuando todavía no se había efectuado. 
Cuarenta y tres años de protestas sociales en Colombia

La Figura 1 muestra las series de protestas sociales y los índices de capacidad del Estado y revela que la contestación social se ha incrementado en el tiempo.

Figura 1. Contestación social y capacidades del Estado, Colombia 1958-2000 (Correlaciones: $r 1=-.46^{* * *}, r 2=-.41^{* *}, N=37$ )

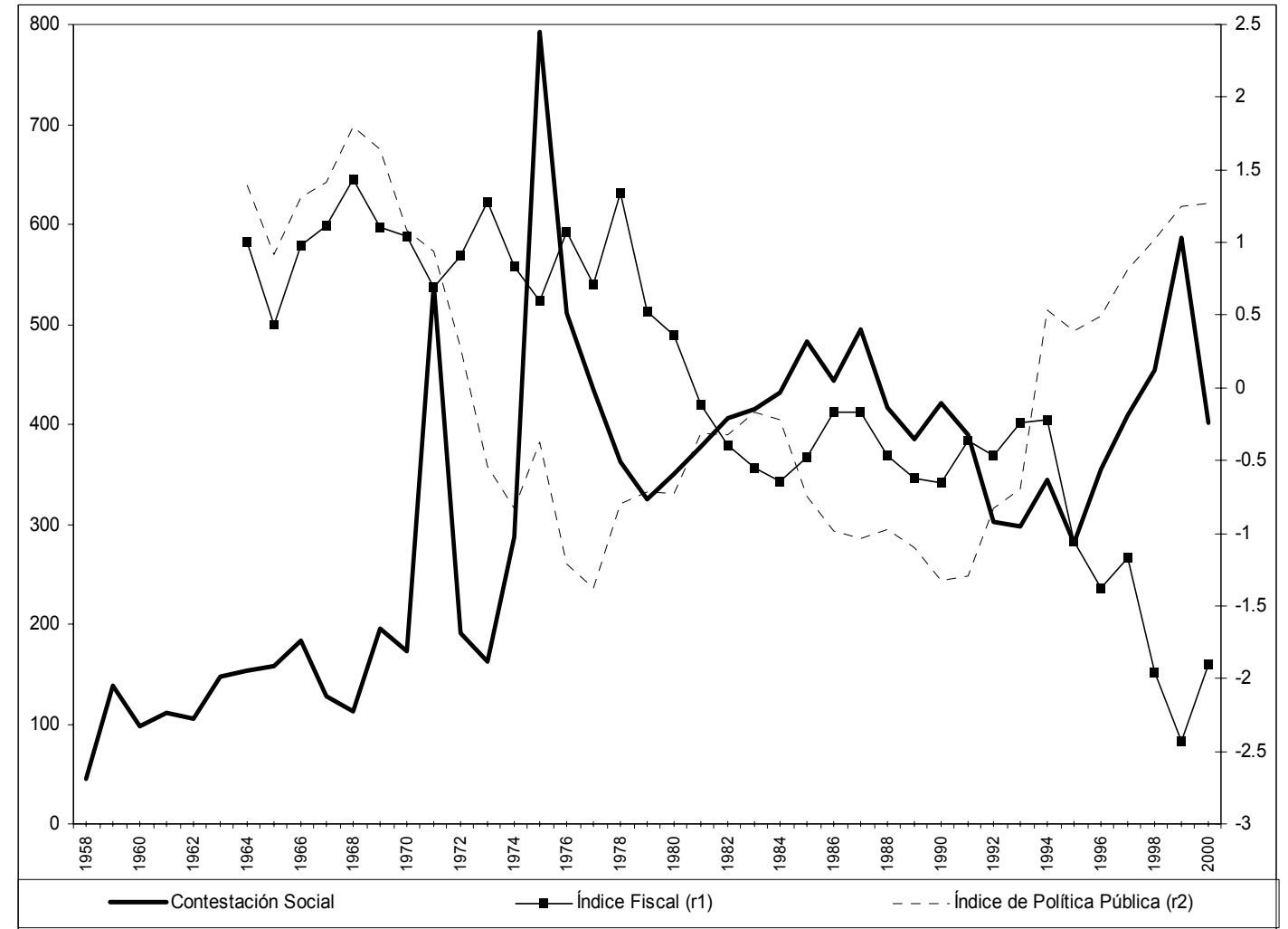

Fuentes: Contestación social Delgado (2003); García (2003a; 2003b; 2003c); Prada (2003); y Archila (2003c). Nota: Prueba de significancia a una cola, índice de correlación de Pearson $p<.01^{* * *}, p^{*} .05^{* *}$, $\mathrm{p}<.1^{*}$

Se observan picos de contestación en 1971, 1975 y 1999 y picos más pequeños en 1985 y 1987. En 1971, se registraron más de 500 actos como resultado de las invasiones campesinas de tierra, el movimiento estudiantil y la actividad sindical en contra de la violación de pactos laborales y por la mejora de sus condiciones de empleo. En 1975, más de 750 acciones fueron registradas debido a la fuerte movilización de los sectores urbanos, incluyendo estudiantes y trabajadores, que protestaron el incumplimiento de las propuestas de apertura política y reforma social prometidas por el gobierno de Alfonso López (1974-1978), 
y contra el incremento de precios y el decrecimiento de los salariales reales. Hacia finales de los setenta, las protestas se reducen como resultado de la represión estatal, amparada bajo el estatuto de seguridad del gobierno de Julio César Turbay (1978-1982), que entre otras cosas, justificó la persecución política de los opositores del gobierno. Se observa luego un crecimiento de la protesta en los años ochenta como resultado de la crisis política, las violaciones de derechos humanos, y la recurrente violación de los derechos laborales, entre otras.

Las acciones contestatarias cayeron durante los primeros años del periodo neoliberal (1990-2000) cuando las instituciones democráticas fueron ajustadas a un contexto de mayor liberalización económica y mayores canales de participación. El promedio de acciones contestatarias durante este periodo se redujo a 380 , aunque en 1999 más de 500 protestas fueron observadas como respuesta a la crisis de desempleo y decrecimiento que afligió a la economía nacional como resultado de la apertura económica y las reformas financieras que quebraron buena parte de la producción y el empleo nacional, y la flexibilización laboral y de los sistemas de seguridad social que hicieron más precarias las condiciones de empleo de los colombianos. Los campesinos se movilizaron junto a algunos gremios para defender la producción agraria afectada por la importación de alimentos, el movimiento laboral protestó las medidas de privatización, los estudiantes se movilizaron en contra del alza de matrículas y los recortes presupuestales, y la clase media se movilizó para defenderse de la crisis del sistema financiero que causó la pérdida de sus viviendas, y muchos colombianos se movilizaron para pedir el respeto de la vida y de los derechos humanos.

Como se demostró arriba, el análisis factorial produjo dos componentes de capacidades del Estado. El primer factor fue denominado el Índice Fiscal y el segundo el Índice de Políticas Públicas. El Índice Fiscal demuestra que las capacidades del Estado colombiano se han desmejorado entre 1964 y el 2000. Este índice es más alto entre 1964 y 1979 cuando el gobierno no estaba tan endeudado, sus déficit fiscales eran manejables, y el conflicto armado era latente ${ }^{14}$. El Índice de Política Pública muestra que las capacidades del Estado eran relativamente más altas entre 1964 y 1971, y más bajas entre 1974 y 1993. Los gobiernos del Frente Nacional (1958-1974) gastaron una proporción más alta de su presupuesto en la modernización de la infraestructura del país y en seguridad. Estos gobiernos mantuvieron índices de inflación relativamente bajos (comparado con otros gobiernos latinoamericanos), y le asignaron porcentajes comparativamente más

\footnotetext{
14 Durante la mayoría del periodo del Frente Nacional (1958 hasta 1973) el déficit fiscal era menor al $1 \%$ del PIB. Después de 1979 los gobiernos han producido déficit de 2.4\%, muchas veces financiados con deuda interna y externa. Las décadas de los setenta y ochentas tuvieron tasas de crecimiento más robustas (promedio de 5\%). El índice baja entre 1980 y 1984 en la medida en que la deuda externa aumenta, el conflicto deja de ser latente, y el crecimiento económico baja.
} 
altos del gasto público a la inversión social. Por su lado, los gobiernos de los noventa redujeron la tasa de inflación y le empezaron a asignar un porcentaje importante de su gasto a la infraestructura y al gasto en justicia y seguridad ${ }^{15}$. Finalmente, las correlaciones muestran una relación inversa entre las capacidades del Estado y la contestación social. Es decir, la contestación se incrementa en la medida en que el Estado pierde capacidades, resultado que corrobora en parte mi hipótesis.

Figura 2. Contestación social y capacidades ciudadanas, Colombia 1958-2000 (Correlación: $r=.29 * *, N=37$ )

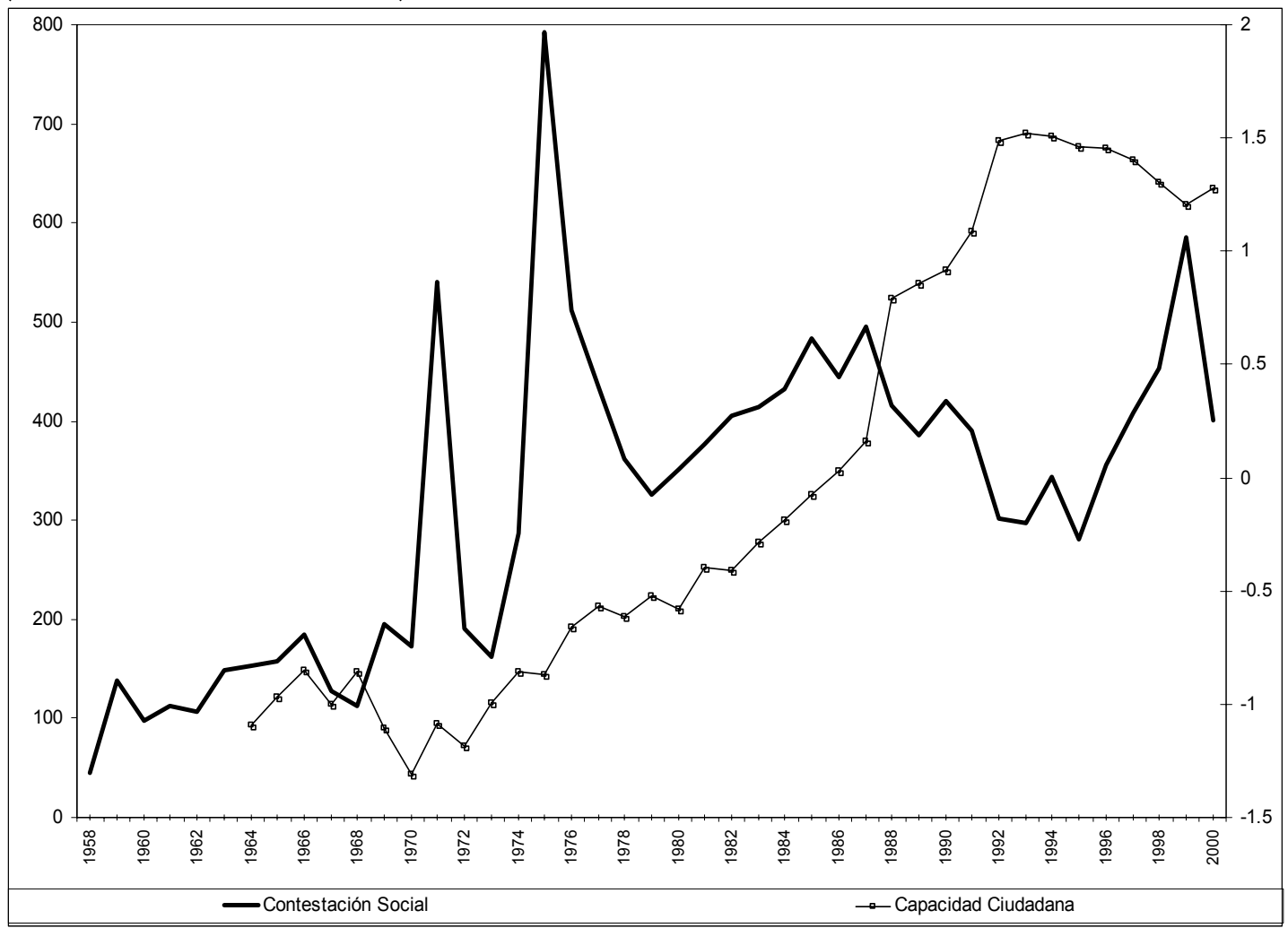

Fuentes: Contestación social Delgado (2003); García (2003a; 2003b; 2003c); Prada (2003); y Archila (2003c). Nota: Prueba de significancia a una cola, índice de correlación de Pearson $p<.01^{* * *}, p^{*} .05^{* *}$, $p<.1^{*}$

\footnotetext{
15 El principal objetivo de los gobiernos neoliberales es lograr la estabilidad macroeconómica para atraer capital financiero. El gasto del gobierno tiende a beneficiar a aquellos sectores más atractivos para los inversionistas, como son las telecomunicaciones y el transporte. El gobierno además está haciendo esfuerzos importantes en el gasto en seguridad y justicia para derrotar la guerrilla y el narcotráfico.
} 
La Figura 2 muestra las series de protestas sociales y el índice de capacidad ciudadana. Las capacidades ciudadanas crecieron gradualmente entre 1970 hasta 1990, y llegaron a su punto más alto entre los años 1991 y 1994 . Varios factores explican el aumento de las capacidades ciudadanas: primero los avances socioeconómicos que aumentaron la esperanza de vida al nacer de la población ${ }^{16}$, segundo la implementación gradual de políticas que optimizaron la relación entre el gobierno y los ciudadanos, y finalmente, el acrecentamiento del empleo en el Estado, la urbanización y el desarrollo de industrias en algunas regiones mejoraron las posibilidades de asociación de los ciudadanos en la medida en que un número mayor de personas trabajaban y vivían en condiciones más organizadas y más susceptibles a las regulaciones del Estado.

El deterioro económico y una tasa de homicidios más alta explican la caída del índice después de 1995. Finalmente, la correlación entre capacidades ciudadanas y contestación social es positiva, lo que desafía mi teoría de que la contestación aumenta en la medida en que bajan las capacidades ciudadanas. En otras palabras, la correlación entre protesta y capacidad ciudadana es positiva si no controlamos estadísticamente el efecto del Estado. Por lo tanto debemos evaluar la relación con una regresión múltiple.

\section{Comprobación de las hipótesis}

Las tres hipótesis de este trabajo - (1) la contestación social aumenta en la medida en que las capacidades del Estado y de los ciudadanos se deterioran, (2) en un contexto de deterioro de las capacidades ciudadanas y del Estado, el paso del tiempo aumenta la contestación social, y (3) la Constitución de 1991 no afectó el comportamiento de la política contestataria - serán examinadas usando los datos del CINEP como medida de la variable dependiente (total de protestas), los factores que construí para medir las capacidades ciudadanas y del Estado, y las variables dicotómicas que miden los regimenes políticos. Estas hipótesis serán evaluadas por medio de regresiones de series de tiempo con corrección de colinealidad utilizando el procedimiento Cochrane-Orcutt y Prais Winsten con la ayuda del programa estadístico STATA. Estas regresiones de tiempo utilizan observaciones nacionales de las variables dependiente e independiente entre los años 1964-200017.

\footnotetext{
16 Por ejemplo, los años de escolaridad han aumentado de 3.1 en 1958 a 7.3 en el 2000. Sin embargo, aunque hayan aumentado, en promedio los colombianos tienen un nivel educativo elemental. EI PIB per capita aumentó gradualmente de $\$ 996$ en 1958 a $\$ 2438$ en 1997, pero un PIB per capita de $\$ 2438$ caracteriza a Colombia como un país con poco desarrollo y condiciones de vida inadecuadas para la población. Finalmente, la expectativa de vida aumentó de 56 años en 1958 a 71 en el 2000.

17 En las ecuaciones de regresión, los coeficientes indican qué tanto cambia la variable dependiente con un incremento de una unidad en la variable independiente, controlando estadísticamente las demás
} 
Tabla 3. Análisis de regresión de series de tiempo. Contestación social en Colombia (1964-2000).

\begin{tabular}{|c|c|c|c|c|c|c|c|c|c|c|c|}
\hline \multicolumn{4}{|c|}{ Hipótesis 1} & \multicolumn{4}{|c|}{ Hipótesis 2} & \multicolumn{4}{|c|}{ Hipótesis 3} \\
\hline Coeficiente & $\begin{array}{c}\text { Nivel de } \\
\text { Significancia }\end{array}$ & & $\begin{array}{c}\text { Beta } \\
\text { Ponderada }\end{array}$ & Coeficiente & $\begin{array}{c}\text { Nivel de } \\
\text { Significancia }\end{array}$ & & $\begin{array}{c}\text { Beta } \\
\text { Ponderada }\end{array}$ & Coeficiente & $\begin{array}{c}\text { Nivel de } \\
\text { Significancia }\end{array}$ & & $\begin{array}{c}\text { Beta } \\
\text { Ponderada }\end{array}$ \\
\hline$\cdot 124,702$ & 0,002 & $* * *$ & $.0,780$ & $.71,402$ & 0,170 & & $.0,447$ & $.72,479$ & 0,197 & & $.0,453$ \\
\hline $.63,383$ & 0,007 & $* * *$ & $.0,396$ & $.48,530$ & 0,048 & $* *$ & $.0,304$ & $.49,781$ & 0,117 & & $.0,311$ \\
\hline \multirow[t]{3}{*}{$.73,648$} & 0,061 & * & $.0,461$ & $.150,574$ & 0,022 & $* *$ & $.0,942$ & $.152,739$ & 0,042 & $* *$ & $.0,955$ \\
\hline & & & & 12,326 & 0,149 & & 0,968 & 12,194 & 0,174 & & 0,958 \\
\hline & & & & & & & & 6,360 & 0,952 & & 0,017 \\
\hline 357,587 & 0,000 & & & 47,443 & 0,823 & & & 49,028 & 0,822 & & \\
\hline 36 & & & & 36 & & & & 36 & & & \\
\hline 6,240 & 0,002 & $* * *$ & & 6,230 & 0,001 & $* * *$ & & 4,860 & 0,002 & & \\
\hline 0,369 & & & & 0,446 & & & & 0,448 & & & \\
\hline 0,310 & & & & 0,374 & & & & 0,355 & & & \\
\hline 1,822 & & & & 1,928 & & & & 1,937 & & & \\
\hline 1,967 & & & & 1,970 & & & & 1,970 & & & \\
\hline
\end{tabular}

Nota: $p<.01^{* * *}, p<.05^{* *}, p<.1^{*}$. La media de las variables dicotómicas corresponde a la proporción (o porcentaje si se multiplica por 100) de los casos que tienen un 1.

variables independientes. La Beta ponderada es el coeficiente estandarizado que muestra la influencia relativa de cada una de las variables independientes sobre la variable dependiente. Entre más grande sea la Beta, más fuerte es la relación de esa variable con la variable dependiente. Finalmente, el nivel de significancia es el criterio que se usa para rechazar una hipótesis nula. Como este es un trabajo exploratorio, usaré un nivel de significancia de .10 como umbral. 
Las regresiones presentadas en la Tabla 3 prueban que todas las hipótesis son válidas y explican entre el $31 \%$ y el $38 \%$ de la variación en la protesta social (ver $\mathrm{R}^{2}$ ajustado) ${ }^{18}$. Además, las ecuaciones tienen poca colinialidad, ya que la estadística Durbin Watson original se acerca mucho a 2. La falta de auto-correlación indica que existe un alto grado de independencia entre las observaciones de todas las variables que introduje en el modelo. También demuestra que los residuos de la ecuación son independientes ya que no están distribuidos de manera sistemática en la ecuación, lo que inflaría el resultado de la regresión.

La ecuación de la hipótesis 1 establece que el deterioro de las capacidades ciudadanas y del Estado aumenta el uso de la política contestataria entre los actores sociales. Sin embargo, la contestación social es mucho más sensible al deterioro de las capacidades del Estado - ya que el nivel de significancia de estas variables está por debajo del 0.01 - y es menos sensible al deterioro de las capacidades ciudadanas. El índice fiscal resulta ser la variable con mayor influencia pues su Beta ponderada es de -0.78.

Por otro lado, la relación negativa que obtuvo el índice de capacidad ciudadana en la regresión comprueba que en un contexto en donde estas capacidades han aumentado (ver Figura 2) se incrementa el uso de la protesta cuando el Estado pierde capacidades. Es decir, los miembros del sistema político esperan seguir mejorando su bienestar político y económico, y al percibir que éste se deteriora como resultado de reveses en las capacidades del Estado para garantizar el bien público, los ciudadanos están más dispuestos a utilizar la contestación como mecanismo de presión política.

La ecuación de la hipótesis 2 introduce la variable de tiempo para evaluar su efecto en la relación entre las variables de capacidad y la contestación social ${ }^{19}$. Esta ecuación demuestra que el efecto del tiempo es estadísticamente insignificante cuando se introduce en la ecuación junto con las variables de capacidad. No obstante lo anterior, la variable del tiempo muestra que la influencia de la capacidad ciudadana y del índice fiscal sobre la contestación social estaba distorsionada por el paso del tiempo. En otras palabras, con la introducción de esta variable a la ecuación, el índice fiscal pierde significancia, el efecto del índice de política pública se mantiene más o menos igual, pero la Beta de capacidad ciudadana aumenta a -0.94 , convirtiéndose en la variable más importante de la ecuación. Esto modifica lo propuesto en la segunda hipótesis del trabajo, donde se

\footnotetext{
18 El $R^{2}$ es el coeficiente de determinación múltiple y es una medida multi-variada de asociación. El R² muestra el grado de variación en la variable dependiente que es explicado por las variables independientes que fueron incluidas en la ecuación. El R ${ }^{2}$ ajustado "encoje" el valor del R² al "penalizar" la inclusión de variables adicionales en la ecuación (O'SULLIVAN Y RASSEL, 1999, p. 440).

19 En las regresiones de series de tiempo, los coeficientes de las variables independientes (en este caso, las variables de capacidad) miden tanto el efecto del paso del tiempo como la variable en sí, a no ser que se introduzca el paso del tiempo como variable independiente.
} 
JARAMILLO, M.V. Cambio institucional e incapacidades institucionales: un análisis...

plantea que en un contexto de pérdida de capacidades, el paso del tiempo aumentará la contestación social. En conclusión, el tiempo no tiene un efecto independiente sobre la contestación.

La tercera hipótesis incluye una variable dicotómica de la Constitución de 1991. Su efecto en la variable dependiente no es significativo estadísticamente. Así, los efectos democratizadores de la nueva constitución-supuestamente diseñados para reducir la contestación social-aparentemente no fueron exitosos. En esta ecuación, el índice fiscal sigue siendo insignificante. Por otro lado, el índice de política pública deja de ser significativo. Así, la variable de capacidad ciudadana continúa siendo la más fuerte.

\section{Conclusiones}

Entre los principales logros de la Constitución de 1991 está la ampliación de los mecanismos de participación ciudadana en los asuntos públicos, y la restricción del uso frecuente de los estados de sitio, limitándolos sólo a situaciones de amenaza a la seguridad nacional. No obstante estos logros para el afianzamiento de los derechos ciudadanos, muchos de los mecanismos de democracia directa contemplados en la constitución son casi inoperantes como resultado de fallas del Estado, y sobretodo, de incumplimiento de acuerdos negociados entre gobierno y ciudadanos. Además, los gobiernos neoliberales han recurrido a prácticas autoritarias como último recurso para implementar medidas poco populares relacionadas con las reformas de mercado, muchas de ellas exigidas por instituciones internacionales como el Fondo Monetario Internacional como parte de los compromisos gubernamentales para acceder a préstamos o por el gobierno de los Estados Unidos para recibir ayuda militar (ver el texto del Plan Colombia).

Por otro lado, las prácticas violentas e iliberales de narcotraficantes, guerrillas y paramilitares han contribuido a deteriorar la institucionalidad democrática de Colombia y a reducir, en amplias zonas del país, la efectividad de los derechos ciudadanos. En otras palabras, la incapacidad estatal de restringir el uso de la violencia contra los miembros del sistema político y la reticencia política del gobierno para hacer que las instituciones de la democracia sean significativas están reduciendo los derechos de los colombianos. Como resultado, muchos ciudadanos organizados deducen que los gobiernos neoliberales, y la Constitución de 1991, sólo contemplan sus derechos en el papel y que las instituciones del país generalmente no representan el bien común. Por lo tanto, no sólo es lógico, sino imperativo, combinar métodos formales y contestatarios para hacer reclamos, formar opinión pública y llevar los debates al gobierno. 
Así, los colombianos han utilizado activamente algunas de las oportunidades políticas contempladas en la Constitución de 1991 para influenciar al gobierno y hacer valer sus derechos, como la tutela o el recurso legal que le da a los ciudadanos acceso rápido a las cortes con el fin de hacer valer derechos fundamentales. También han encontrado aliados en la Corte Constitucional, la Defensoría del Pueblo, e inclusive en la Fiscalía y la Contraloría, instituciones que en ocasiones han actuado a favor de asociaciones de ciudadanos al controvertir procesos de privatización o denunciar violaciones a los derechos humanos. Así, el recurso ciudadano a las tutelas, las acciones populares y a otros mecanismos de participación ha aumentado, a la vez que el número de protestas se mantiene alto.

En general, este trabajo esperaba encontrar que la contestación aumenta en la medida en que las capacidades ciudadanas y del Estado se disminuyen. Este fue un resultado anticipado, pues la pérdida de capacidades del Estado se traduce en inseguridad socioeconómica y política para la gente en general. Consecuentemente, cuando los miembros de sistemas democráticos - onde la promesa de la ciudadanía es percibida como una realidad alcanzable - no tienen acceso a recursos y han perdido derechos utilizarán mecanismos contestatarios para hacerle reclamos al Estado, si los mecanismos legales son inefectivos para manifestar sus preferencias de producción y consumo, sus expectativas de participación política y sus deseos de mejorar su calidad de vida. Con este trabajo he demostrado que mi proposición tiene validez para el caso de Colombia y he usado el análisis de regresiones de tiempo como método empírico para comprobar mi hipótesis. 


\section{Referencias}

ARCHILA, M. Colombia 1975-2000: de crisis en crisis. Contexto. En: ARCHILA, M. et al. 25 Años de luchas sociales en Colombia, Bogotá: CINEP, 2003 a.

Balance de 25 años de luchas en Colombia: Conclusión. En: ARCHILA, M. et al. 25 Años de luchas sociales en Colombia, Bogotá: CINEP, 2003 b.

. Idas y venidas, vueltas y revueltas: Protestas sociales en Colombia 1958-1990.

Bogotá: CINEP, ICANH, 2003 c.

BONILLA, R. y González J. Bien-estar y macroeconomía. Informe de coyuntura. Bogotá: CID, 2003.

BOTTOMORE, T. Ciudadanía y clase social, cuarenta años después. En: MARSHALL Y

BOTTOMORE T., Ciudadanía y clase social. Madrid: Alianza Editorial, 1992.

DELGADO, A. Las luchas laborales. En: ARCHILA, M. et al. 25 Años de luchas sociales en Colombia. Bogotá: CINEP, 2003.

DNP (DEPARTAMENTO NACIONAL DE PLANEACIÓN. UNIDAD DE ANÁLISIS

MACROECONÓMICO). Estadísticas históricas de Colombia. Bogotá: DNP, 1998.

Estadísticas históricas de Colombia, 2002. En:

<http://www.dnp.gov.co/03_PROD/PUBLIC/1p_ee.asp\#3>

Sistema de Indicadores Sociodemográficos para Colombia. En:

<http://www.dnp.gov.co/01_CONT/INDICADO/SISD.HTM>. Consultado en nov. 2004.

ECKSTEIN, S. Power and Popular Protest in Latin America. En: Power and Popular Protest: Latin American Social Movements, Berkeley: University of California Press, 1989.

EDWARDS, S. y STEINER R. On the Crisis Hypothesis of Economic Reform: Colombia 1989. 1991. En: Cuadernos Económicos, n 112, p. 1-51, 2000.

EVANS, P. B., RUESCHEMEYER, D. y SKOCPOL, T. On the Road Toward a More Adequate Understanding of the State. En: EVANS, P. B., RUESCHEMEYER, D. y SKOCPOL, T. (Org.) Bringing the State Back In. Cambridge: Cambridge University Press, 1985. 
FERGUSON, T. The Golden Rule: The Investment Theory of Party Competition and the Logic of Money-Driven Political Systems. Chicago: University of Chicago Press, 1995.

FOWERAKER, J. Theorizing Social Movements. London and Boulder: Pluto Press, 1995.

FOWERAKER, J. y LANDMAN, T. Citizenship Rights and Social Movements: A Comparative and Statistical Analysis. Oxford: Oxford University Press, 1997.

GARCÍA, M. Luchas urbano-regionales. En: 25 Años de luchas sociales en Colombia, ARCHILA, M. et al. Bogotá: CINEP, 2003a.

. Luchas estudiantiles. En: ARCHILA, M. et al. 25 Años de luchas sociales en

Colombia. Bogotá: CINEP, 2003b.

Luchas sociales protagonizadas por actores menos visibles." En: ARCHILA, M. et al. 25 Años de luchas sociales en Colombia. Bogotá: CINEP, 2003c.

GARRETÓN, M. Cambios sociales, actores y acción colectiva en América Latina. Serie Políticas Sociales. Santiago: Cepal, 2001.

GILHODES, P. Las luchas agrarias en Colombia. Bogotá: Ediciones el Tigre de Papel, 1970.

GRINDLE, M. Challenging the State: Crisis and Innovation in Latin America and Africa. Cambridge University Press, 1999.

HUBER, E. Assessments of State Strength. En: SMITH, P.(ed.). Latin America in Comparative Perspective: New Approaches to Methods and Analysis. Boulder: Westview, 1995.

JENKINS, C. Social Movements, Political Representation, and the State: An Agenda and Comparative Framework. In: The Politics of Social Protest: Comparative Perspectives on States and Social Movements. Minneapolis: University of Minnesota Press, 1995.

LEAL, F. Los movimientos políticos y sociales: Un producto de la relación entre Estado y sociedad civil. Análisis político 13, may-ago. 1991.

MARSHALL, T. Citizenship and Social Class. Cambridge: Cambridge University Press. 1950. 
MCADAM, D., TARROW. S.y TILLY, C. Dynamics of Contention. Cambridge: Cambridge University Press, 2001.

O’DONNELL, G. Delegative Democracy. Journal of Democracy, 5(1), p. 55-69, 1994.

O'SULLIVAN, E. y RASSEL, G. Research Methods for Public Administrators. New York: Longman, 1999.

PÉCAUT, D. Política y sindicalismo en Colombia. Bogotá: Ediciones Culturales, 1982.

PIVEN, F. y CLOWARD, R. Poor People's Movements: Why They Succeed, How They Fail. New York: Vintage, 1977.

PRADA, E. Luchas Campesinas e indígenas. En: ARCHILA, M. et al. 25 Años de luchas sociales en Colombia. Bogotá: CINEP, 2003.

RAMÍREZ, M. Los movimientos cívicos como movimientos sociales en el Putumayo: el poder visible de la sociedad civil. En: ARCHILA, M. y PARDO, M. Movimientos sociales, Estado y democracia en Colombia. Bogotá: CES/Universidad Nacional, ICANH, 2001 a.

M. Entre el Estado y la guerrilla: identidad y ciudadanía en el movimiento de los campesinos cocaleros del Putumayo. Bogotá: Colciencias, ICANH, 2001b.

RUESCHEMEYER, D. et al. Capitalist Development \& Democracy. Chicago: University of Chicago Press, 1992.

SANTANA, P. Desarrollo regional y paros cívicos en Colombia. Controversia 107-108. Bogotá: Cinep, 1983. 47.61, 1988.

P. Los movimientos cívicos. El nuevo fenómeno electoral. En: Revista Foro Nº6, p.

P. Crisis municipal: movimientos sociales y reforma política en Colombia. En: Revista Foro No 1, p. 4-15, 1986.

SEN, A. Development as Freedon. New York: Random House, 1999. 
SKOCPOL, T. Bringing the State Back In: Strategies of Analysis in Current Research. In:

SKOCPOL, T. (ed.).Bringing the State Back in. Cambridge: Cambridge University Press, 1985.

STEINMO, S. e THELEN, K. Historical Institutionalism in Comparative Politics. In: STEINMO, T. et al (eds.). Structuring Politics: Historical Institutionalism in Comparative Analysis.

Cambridge and New York: Cambridge University Press, 1992.

TARROW, S. Social Movements in Contentious Politics: A Review Article. The American Political Science Review, 90 (4), p. 874-883, 1996.

S. Power in Movement: Social Movements and Contentious Politics. Second Edition. Cambridge: Cambridge University Press, 1998.

THELEN, K. How institutions evolve: insights from comparative-historical analysis. In: MAHONEY, J. (ed.). Comparative Historical Analysis in the Social Sciences. Cambridge: Cambridge University Press, 2003.

TILLY, C. Introduction. In: TILLY, L. and TILLY, C. (eds.). Class Conflict and Collective Action. Beverly Hills and London: Sage Publications, 1981.

Social Movements and National Politics. In: BRIGHT, C. and HARDING, S (eds). Statemaking and Social Movements. Ann Arbor: University of Michigan, 1984.

.War Making and State Making as Organized Crime. In: SKOCPOL, T. et al (ed.). Bringing the State Back in. Cambridge: Cambridge University Press, 1985.

. Citizenship, Identity and Social History. In: TILLY, C. (ed). Citizenship, Identity and Social History. Cambridge: Cambridge University Press, 1996.

TOURAINE, A. Las sociedades dependientes: Ensayos sobre América Latina. México: Siglo Veintiuno Editores, 1976.

The Voice and the Eye: An Analysis of Social Movements. Cambridge: Cambridge University Press, 1985.

The Return of the Actor: Social Theory in Post-Industrial Society. Minneapolis: University of Minnesota Press, 1988.

URRUTIA, M. Development of the Colombian Labor Movement. New Haven: Yale University, 1969. 
VILAS, C. Después del ajuste: La política social entre el Estado y el mercado. En: VILAS, C. (ed.). Estado y políticas sociales después del ajuste. Caracas: Nueva Sociedad, 1995.

WEISS, L. States in the Global Economy. Cambridge, 2003.

WILKINSON, P. Social Movements. London: Macmillan, 1971.

WORLD BANK. World Development Indicators CD-ROM. Washington, DC: The World Bank, 2002.

ZAMOSC, L. Peasant Struggles of the 1970s in Colombia. In: ECKSTEIN, S. (ed) Power and Popular Protest: Latin American Social Movements. Berkeley, University of California Press, 1989. 